\title{
EVOLUCIÓN FINANCIERA DE LA BANCA MÚLTIPLE PERUANA 2007-2017
}

\author{
LOURDES EMMERICH* \\ Universidad de Lima, Perú
}

Recibido: 19 de agosto del 2019 / Aprobado: 16 de octubre del 2019

doi: $10.26439 /$ ing.ind2020.n038.4818

RESUMEN: El desempeño financiero de la banca múltiple (BM) peruana entre 2007 y 2017 ha mostrado que esta presenta una alta concentración bancaria, solvencia, rentabilidad y liquidez. Se analizan las variables como capital, activos, gestión, rentabilidad y liquidez. Las oportunidades de mejora de la banca múltiple peruana se centran en la inclusión financiera y economías de escala enfrentando los riesgos de volatilidad, vulnerabilidad ante fenómenos climatológicos y la corrupción política.

Palabras clave: Perú / bancos / finanzas / historia económica / intermediación financiera

\section{PERUVIAN BANKING FINANCIAL PERFORMANCE 2007-2017}

ABSTRACT: The financial performance of Peruvian banks from 2007 to 2017 has shown high banking concentration, solvency, profitability and liquidity. This research analyzes variables such as capital, assets, management, profitability and liquidity. The improvement opportunities in Peruvian banks are focused on financial inclusion and economies of scale, in view of volatility and vulnerability risks to climate phenomena and political corruption.

Keywords: Peru / banks / finance / economic history / financial intermediation 


\section{INTRODUCCIÓN}

La banca múltiple (BM) juega un papel esencial en la distribución de los recursos a través de la intermediación financiera y en el desarrollo económico del país (Jeanneau, Tovar y Moreno, 2007). El desarrollo del sistema financiero tiene influencia en el crecimiento económico de una nación (Levine, 2005) por lo que el propósito del presente estudio es evaluar el desempeño de la banca múltiple peruana durante el oncenio del 2007 al 2017. Este período abarca eventos económicos relevantes como antes y durante el auge de la crisis subprime y su recuperación, el crecimiento económico del Perú y la desaceleración actual.

El desarrollo financiero es un buen indicador del crecimiento económico. Un mayor desarrollo de los servicios bancarios, el manejo del riesgo y la información, propicia el crecimiento económico (Galarza y Miranda, 1995). El desarrollo económico comúnmente ha sido asociado a términos como riqueza, trabajo, ingresos. Sin embargo, el desarrollo también involucra al aspecto financiero igual que los bienes y servicios (Gurley y Shaw, 1955).

Mientras que el Banco Central de Reserva del Perú (BCRP), a través de la implementación de la política monetaria, impacta la oferta de crédito (Gómez Alberca, Villanueva y Erixon, 2018), el desarrollo del mercado crediticio también puede afectar significativamente al desarrollo económico de una región (Hansen y Sulla, 2013).

El sistema financiero (SF) peruano está compuesto por la banca múltiple (BM), financieras y microfinancieras y empresas de arrendamiento financiero. Las microfinancieras incluyen a las cajas municipales (CM), cajas rurales de ahorro y crédito (CRAC) y las entidades de desarrollo de la pequeña y microempresa (Edpymes). A diciembre 2018, el sistema financiero peruano se componía de 55 empresas, 16 pertenecientes a la banca múltiple, 11 empresas financieras, 12 cajas municipales, 6 cajas rurales de ahorro y crédito, 9 entidades de desarrollo de la micro y pequeña empresa y 1 empresa de arrendamiento financiero. En el sistema financiero peruano actual, el 89,28 \% de los activos totales se concentra en la banca múltiple. Más del $50 \%$ de los créditos directos corresponden a créditos de corporaciones a grandes y medianas empresas. Un poco más del $20 \%$ de los créditos directos corresponden a créditos de consumo, mientras que la cartera hipotecaria representa el $15 \%$ de los créditos directos. Finalmente, el $13 \%$ de los créditos directos se dirigen a micro y pequeñas empresas (Superintendencia de Banca, 2018).

\section{METODOLOGÍA}

El estudio comprende el análisis evolutivo financiero de las variables propuestas por la metodología CAMEL (capital, assets, management, earnings, liquidity), incluyendo 
comparaciones con la banca múltiple de otros países latinoamericanos (Argentina, Bolivia, Brasil, Chile, Colombia, Ecuador, México, Panamá, Paraguay y Uruguay), con el fin de entender la dimensión y potencial del negocio. Adicionalmente, se analiza la correlación de variables.

Tabla 1

Metodología para el análisis financiero de la BM peruana período 2007-2017

\begin{tabular}{|c|c|c|c|}
\hline & Grupo de análisis & Rubro analizado & Análisis realizado \\
\hline \multirow[t]{8}{*}{ C } & Capital - Capital & $\begin{array}{l}\text { Concentración } \\
\text { bancaria }\end{array}$ & $\begin{array}{l}\text { Evolución de la concentración bancaria y la cantidad } \\
\text { de bancos }\end{array}$ \\
\hline & & & Concentración bancaria a nivel de activos \\
\hline & & & $\begin{array}{l}\text { Evolución de la participación de activos de los pri- } \\
\text { meros cinco bancos en el ranking de participación }\end{array}$ \\
\hline & & Solvencia & Capital / activos ponderados por riesgo (APR) \\
\hline & & & Evolución de la solvencia \\
\hline & & & Evolución del patrimonio \\
\hline & & & $\begin{array}{l}\text { Variación porcentual del PBI y variación porcentual } \\
\text { del patrimonio }\end{array}$ \\
\hline & & & $\begin{array}{l}\text { Análisis cualitativo del respaldo patrimonial de cada } \\
\text { banco }\end{array}$ \\
\hline \multirow[t]{13}{*}{ A } & Assets - Activos & Análisis de activos & Evolución de los activos, pasivos y patrimonio \\
\hline & & & Evolución de activos \\
\hline & & & Variación porcentual del PBI y los activos de la BM \\
\hline & & & $\begin{array}{l}\text { Coeficiente de correlación entre la variación porcen- } \\
\text { tual del PBI y los activos de la BM }\end{array}$ \\
\hline & & & $\begin{array}{l}\text { Volumen de los activos de la BM peruana en compa- } \\
\text { ración con otros países de la región }\end{array}$ \\
\hline & & Morosidad & Morosidad \\
\hline & & & $\begin{array}{l}\text { La morosidad de la BM peruana en comparación } \\
\text { con otros países de la región }\end{array}$ \\
\hline & & & Correlación entre la morosidad y la rentabilidad \\
\hline & & & $\begin{array}{l}\text { Morosidad y rentabilidad en comparación con otros } \\
\text { países de la región }\end{array}$ \\
\hline & & & Evolución del ROE y la morosidad \\
\hline & & & Morosidad por tipo de crédito \\
\hline & & & Evolución del tipo de cambio y la morosidad \\
\hline & & Cobertura & Provisiones / cartera atrasada \\
\hline
\end{tabular}


(continuación)

\begin{tabular}{lll}
\hline M Management-Gestión & Eficiencia & Eficiencia \\
& Eficiencia de gastos de administración \\
& Eficiencia de gastos de operación \\
& Eficiencia de personal \\
& Eficiencia de oficinas \\
\hline E Earnings & Rentabilidad & Rentabilidad en comparación con otros países de la \\
& & región \\
& & Evolución de la rentabilidad sobre el capital (ROE) \\
& & Evolución de la rentabilidad sobre los activos (ROA) \\
& Correlación entre el índice de precios al consumidor \\
& (IPC) y la rentabilidad (ROE y ROA) \\
& Evolución de las tasas activas y pasivas por moneda \\
& Margen financiero & Evolución del spread \\
\hline Liquidity - Liquidez & Liquidez & Coeficiente de dolarización de la liquidez \\
\hline
\end{tabular}

Elaboración propia

\section{RESULTADOS}

\subsection{Concentración bancaria}

La concentración bancaria de los cinco bancos con mayor participación de activos en la $\mathrm{BM}$ ha tenido el siguiente comportamiento durante el período de estudio.

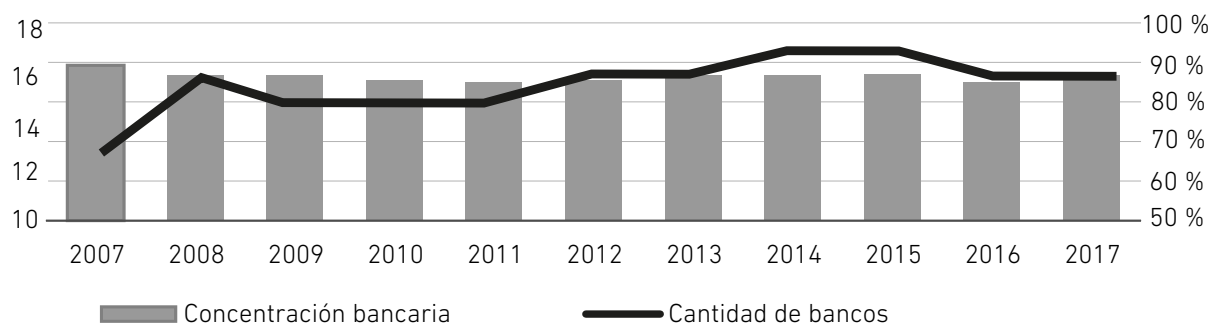

Figura 1. Evolución de la concentración bancaria y cantidad de bancos en el Perú

Fuente: Superintendencia de Banca, Seguros y AFP (SBS, 2018a)

Elaboración propia 
La concentración bancaria (CB), a nivel de activos, se mantuvo durante el período de estudio en un promedio de $86,92 \%$. La CB se afectó significativamente por las fusiones, adquisiciones, entradas y salidas de participantes con alto número de activos en el mercado. Períodos donde pequeños bancos ingresaron o se retiraron del mercado manteniendo la concentración bancaria relativamente estable.

De hecho, en el año 1998, cuando existían en el Perú 25 bancos, la CB era de 67,35\%, pero dos años después (2000) participaron en la BM sólo 16 bancos; las 2 entidades que representaban el $10 \%$ del total de activos fueron absorbidas por el Banco Wiese (ahora Scotiabank) y las 7 entidades que representaban el 11,5\% del total de activos del mercado fueron liquidadas. Como resultado la CB alcanzó más del $80 \%$ en tan solo dos años. En los siguientes años, del 2000 al 2006, la BM registró la salida de cinco bancos, unos fueron liquidados y otros absorbidos por las entidades más grandes, haciendo que la CB llegara a su pico más alto $(89,6 \%)$ con tan solo 11 participantes.

Sin embargo, entre los años 2006 al 2008 hubo un nuevo panorama. Otros participantes entraron al mercado. Llegó Scotiabank (Canadá) a tomar el control del Banco Wiese, que ya se posicionaba como el tercer banco en participación de activos. Ingresaron los bancos Falabella, Ripley, HSBC, Azteca y Santander. Nuevamente, la BM contaba con 16 entidades. En los siguientes años la CB continuó siendo más del $87 \%$. Los bancos más grandes continuaron creciendo absorbiendo a entidades y comprando carteras de otros bancos más pequeños. Al final del 2017, la BM contaba con 16 entidades y la CB era del $86,8 \%$.

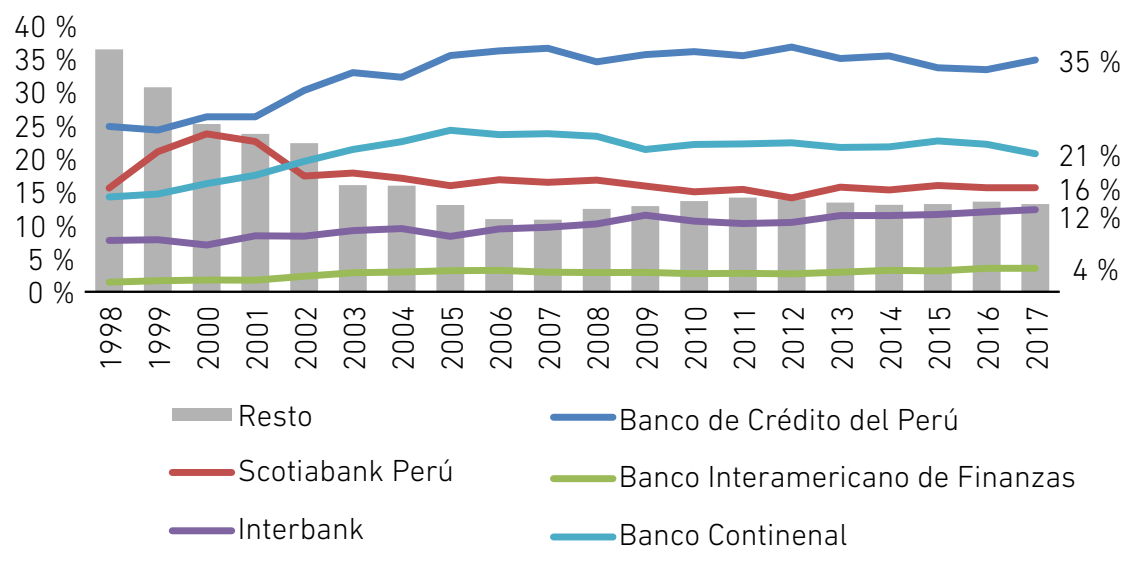

Figura 2. Evolución del porcentaje de participación de activos en 5 bancos

Fuente: SBS (2018b)

Elaboración propia 


\subsection{Solvencia}

Los estándares internacionales plantearon un requerimiento de capital mínimo del 8 \% desde el 2007. El promedio de la relación del capital entre los APR de los miembros de la Federación Latinoamericana de Bancos (FELABAN) era del $15 \%$ a diciembre 2017, mientras que el Perú mostraba un índice del 15,33 \%. En ambos casos, son los índices más altos alcanzados durante el período de estudio.

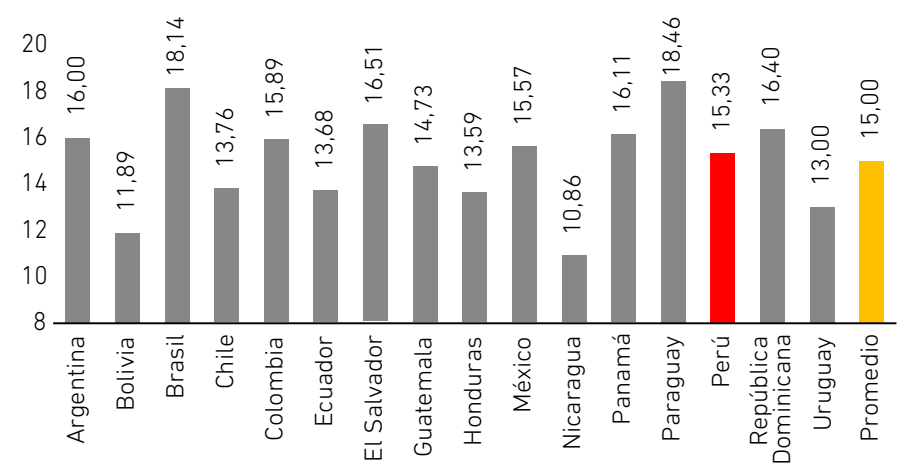

Figura 3. Latinoamérica. Porcentaje de capital/activos ponderados por riesgo en el 2017.

Fuente: FELABAN (2018)

Elaboración propia

En el 2009, la SBS implementó un requerimiento mínimo de capital del 9,1\%, el que posteriormente aumentó a 9,5\%, para finalmente requerir un capital mínimo del $10 \%$ sobre el total de activos y contingentes ponderados por riesgo (APR) a partir de julio del 2011.

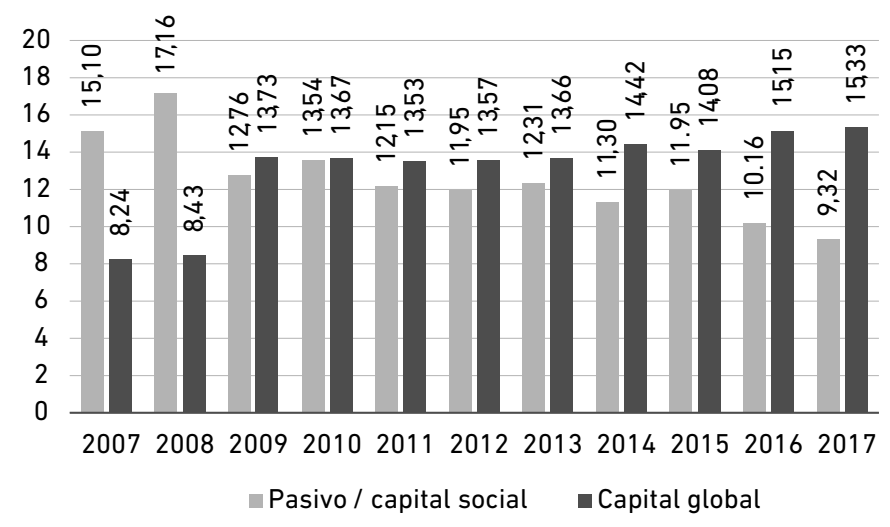

Figura 4. Evolución de la solvencia de la banca múltiple del Perú del 2007 al 2017

Fuente: SBS (2018b)

Elaboración propia 
El patrimonio total de la BM en el Perú aumentó desde el año 2007. El patrimonio del sector se fortaleció durante el período de estudio acompañado de un crecimiento importante de las colocaciones y de los activos. Desde el año 2007, el patrimonio de la banca registró variaciones positivas, con crecimientos anuales de 3,4 mil millones de soles anuales. Mientras que en el año 2007 el patrimonio fue de 9,5 mil millones de soles. A diciembre del 2017, el patrimonio total de la BM peruana alcanza 43,6 mil millones de soles. En promedio, la tasa de crecimiento anual del patrimonio fue del $17 \%$ anual. Sin embargo, la tendencia de crecimiento del patrimonio fue descendente, al igual que el crecimiento económico del Perú. Mientras que en el 2007 el patrimonio total de la BM en el Perú crecía por encima del 20 \%, a diciembre del 2017 el patrimonio aumentó 11 \%.

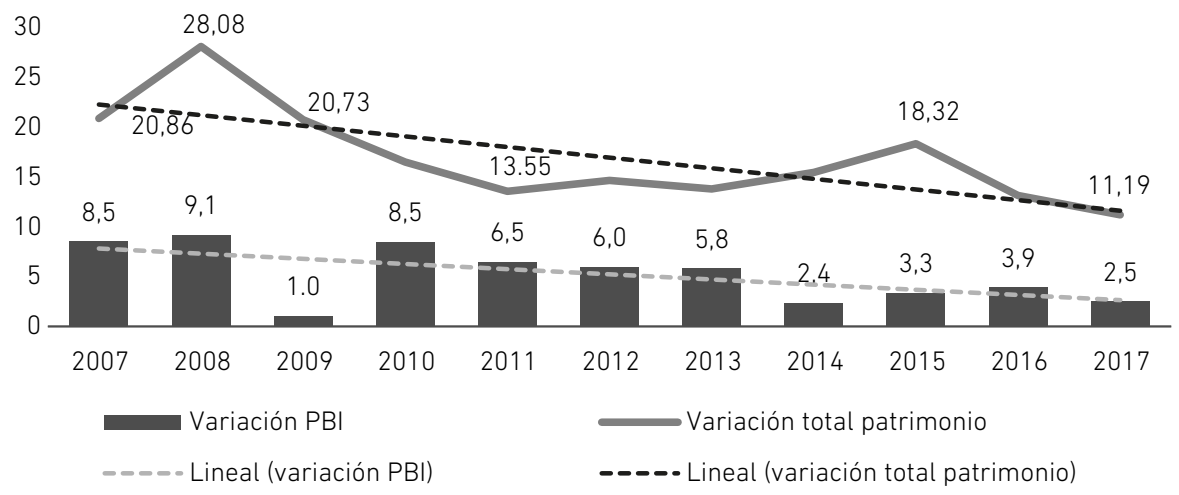

Figura 5. Variación porcentual del PBI versus variación porcentual del patrimonio de la banca múltiple del Perú

Fuente: SBS (2018b) y WorldBank (2018)

Elaboración propia

Tabla 2

Análisis cualitativo de la solvencia de la BM peruana por entidad

\begin{tabular}{ll}
\hline \multicolumn{1}{c}{ Entidades } & \multicolumn{1}{c}{ Análisis cualitativo de la banca múltiple peruana } \\
\hline Banco Azteca & Respaldo patrimonial del Grupo Elektra S.A.B. perteneciente al Grupo Salinas. \\
Banco Cencosud & Respaldo patrimonial del Grupo Cencosud (importante retailer). \\
Banco de Comercio & $\begin{array}{l}\text { Muestra el ratio de capital global más bajo del sistema bancario, representando una } \\
\text { limitación para el crecimiento de la entidad, principalmente, debido a que su mayor } \\
\text { accionista, la Caja de Pensiones Militar Policial, se encuentra limitada de realizar } \\
\text { aportes de capital de manera directa al banco. }\end{array}$ \\
Banco de Crédito del & $\begin{array}{l}\text { Mantiene rigurosos requerimientos de capital por parte del Grupo Credicorp Ltd. } \\
\text { Perú (BCP) }\end{array}$ \\
& $\begin{array}{l}\text { El Grupo Credicorp es una de las empresas holding más importantes del Perú con } \\
\text { participación mayoritaria en empresas como BCP, Prima AFP, Mibanco, Pacífico } \\
\text { Seguros, entre otros. }\end{array}$ \\
\hline
\end{tabular}


(continuación)

\begin{tabular}{|c|c|}
\hline Banco GNB Perú & $\begin{array}{l}\text { Respaldo patrimonial de su accionista controlador Banco GNB Sudameris de } \\
\text { Colombia, holding del Grupo GNB con operaciones en Colombia, Paraguay y Perú. } \\
\text { El patrimonio efectivo es fortalecido por aportes de capital, capitalización de utilida- } \\
\text { des, constitución de reservas y emisión de deuda subordinada. }\end{array}$ \\
\hline $\begin{array}{l}\text { Banco } \\
\text { Interamericano de } \\
\text { Finanzas }\end{array}$ & $\begin{array}{l}\text { Forma parte del grupo de empresas de la familia Fierro (Grupo Fierro), conglomera- } \\
\text { do empresarial con presencia en España, Estados Unidos, Centroamérica y América } \\
\text { del Sur, con inversiones en diversos sectores económicos. }\end{array}$ \\
\hline $\begin{array}{l}\text { Banco Falabella } \\
\text { Perú S.A. }\end{array}$ & Respaldo patrimonial del Grupo Falabella. \\
\hline Banco Pichincha & $\begin{array}{l}\text { Respaldo patrimonial del Grupo Pichincha de Ecuador, cuyo principal activo es el } \\
\text { Banco Pichincha, la institución bancaria más importante del Ecuador. }\end{array}$ \\
\hline $\begin{array}{l}\text { Banco Ripley Perú } \\
\text { S.A. }\end{array}$ & Respaldo patrimonial del Grupo Ripley Corp. \\
\hline Banco Santander & Respaldo patrimonial del Banco Santander de España. \\
\hline BBVA Continental & $\begin{array}{l}\text { Su principal accionista es el BBVA (Banco Bilbao Vizcaya Argentaria), grupo finan- } \\
\text { ciero de prestigio internacional, cuenta con el respaldo patrimonial del grupo de } \\
\text { capital peruano Breca, uno de los más grandes del Perú. }\end{array}$ \\
\hline $\begin{array}{l}\text { Citibank del Perú } \\
\text { S.A. }\end{array}$ & $\begin{array}{l}\text { Pertenece al grupo económico Citigroup Inc. (constituida en Estados Unidos) con } \\
\text { aproximadamente } 200 \text { millones de cuentas abiertas en más de } 160 \text { países. }\end{array}$ \\
\hline ICBC PERU BANK & $\begin{array}{l}\text { Respaldo patrimonial de su accionista mayoritario, Industrial and Commercial Bank } \\
\text { of China Ltd. }\end{array}$ \\
\hline Interbank & Respaldo del Grupo Intercorp. \\
\hline $\begin{array}{l}\text { Mibanco Banco de la } \\
\text { Microempresa S.A. }\end{array}$ & $\begin{array}{l}\text { Respaldo patrimionial del Grupo Credicorp. Absorbe el bloque patrimonial de } \\
\text { Financiera Edyficar. }\end{array}$ \\
\hline $\begin{array}{l}\text { Scotiabank del Perú } \\
\text { S.A. }\end{array}$ & $\begin{array}{l}\text { Respaldo patrimonial del The Bank of Nova Scotia (BNS). Ha ido creciendo debido a } \\
\text { la capitalización parcial de sus utilidades en mayo del } 2018 \text {. }\end{array}$ \\
\hline
\end{tabular}

Fuente: Equilibrium Clasificadora de Riesgos S.A. (2018)

Elaboración propia

\subsection{Análisis de activos}

La banca múltiple peruana, en los últimos 11 años, registró un crecimiento del $242 \%$, triplicando el monto de los activos. El crecimiento promedio anual fue del 15,17 \%. Entre los años 2007 y 2010 , el crecimiento promedio anual de los activos de la BM peruana fue en promedio $22,44 \%$. 
400000

350000

300000

250000

200000

150000

100000

50000

0

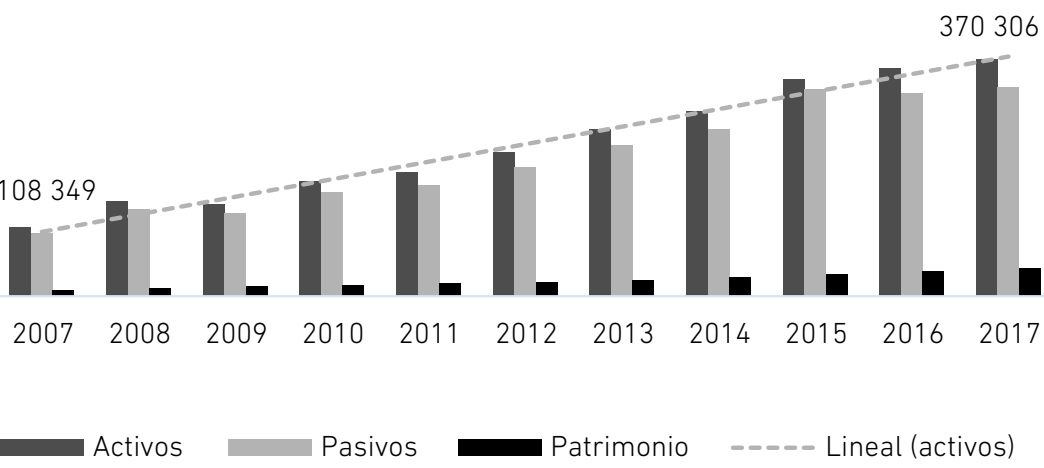

Figura 6. Perú. Evolución de activos, pasivos y patrimonio de la BM peruana

Fuente: SBS (2018)

Elaboración propia

En los siguientes cinco años, del 2011 al 2015, los activos crecieron un promedio anual del 13,60 \%. En el año 2012, los activos de la BM se duplicaron. Sin embargo, en los dos últimos años, 2016 y 2017, los activos de la BM peruana aumentaron tan solo un promedio del 4,54 \% anual, el crecimiento más bajo desde el año 2004 (excluyendo la disminución durante la crisis subprime), observándose una desaceleración del crecimiento de los activos totales.

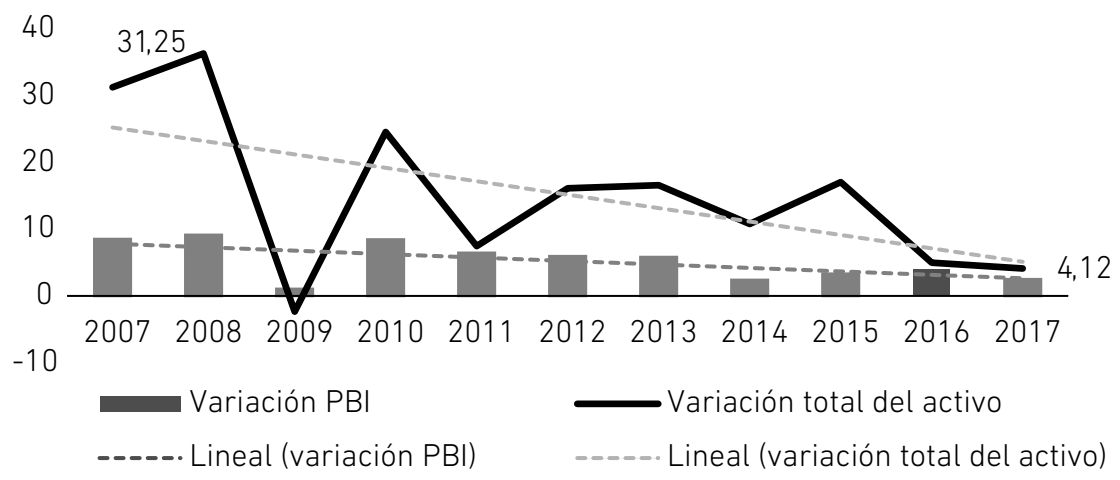

Figura 7. Variación porcentual del PBI versus variación porcentual de los activos de la banca múltiple en el Perú, 2007-2017

Fuente: SBS (2018)

Elaboración propia 
El coeficiente de correlación entre la variación porcentual del PBI y los activos de la $\mathrm{BM}$ peruana en el período de estudio es del $86 \%$; a menor crecimiento del $\mathrm{PBI}$, menor crecimiento de activos.

A diciembre del 2017, los activos de la BM del Perú ascendían a 114 mil millones de dólares, cifra cinco veces mayor que Paraguay, cuatro veces mayor que Bolivia y tres veces mayor que Ecuador. Al comparar los activos de la BM del Perú con economías más grandes, como Brasil y México, se observó que el total de los activos de la BM de Brasil era veinte veces más grande que la peruana y de México cuatro veces, seguido por la de Chile con tres veces.

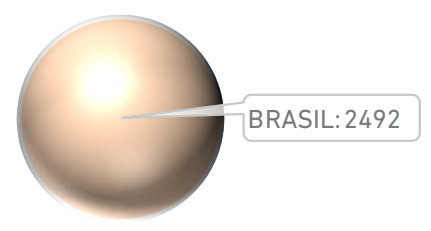

- ARGENTINA

• BOLIVIA

BRASIL

- CHILE

- COLOMBIA

- ECUADOR

• MÉXICO

- PANAMÁ

- PARAGUAY

๑) PERÚ

- URUGUAY

Figura 8. Activos de la banca múltiple en miles de millones de dólares a diciembre del 2017 en Latinoamérica

Fuente: Felaban (2018)

Elaboración propia

\subsection{Morosidad}

A diciembre del 2017, en el Perú, el 3 \% de la cartera se encontraba atrasada. La morosidad registró un aumento paulatino durante el período de estudio.

Comparando el porcentaje de morosidad con el resto de los países latinoamericanos, Perú estuvo por debajo de Brasil (5,60 \%), Colombia (4,15\%), Ecuador (3,45 \%) y Paraguay 
$(3,20 \%)$, registrando una morosidad del 3,09\%. El resto de los cuatro países con los menores índices de morosidad registraron un promedio de $1,86 \%$. Se puede concluir que la BM peruana mantuvo un alto índice de rentabilidad y un indicador de morosidad saludable dentro del promedio de la región, sin embargo, la calidad de la cartera mostró un deterioro paulatino a través del tiempo con una la rentabilidad sobre el patrimonio (ROE) en descenso.

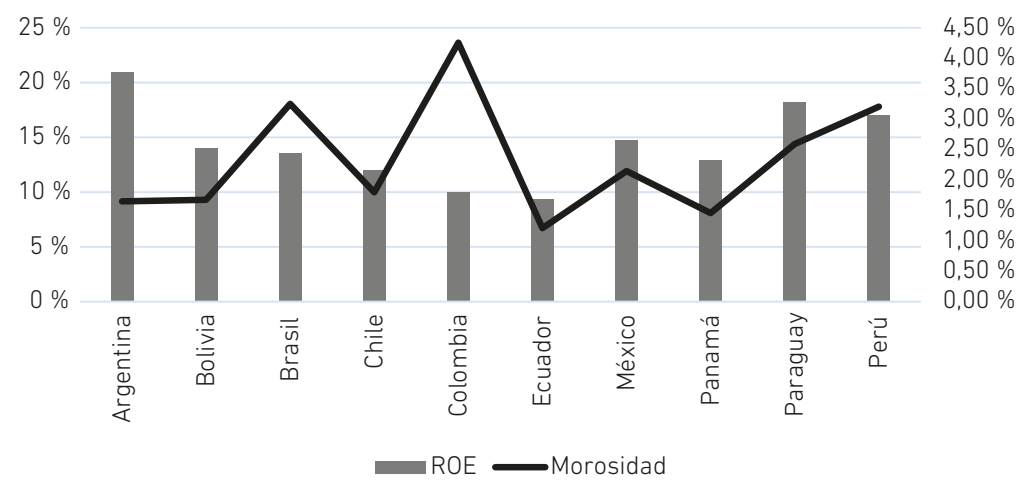

Figura 9. Rentabilidad y morosidad a diciembre del 2017 en Latinoamérica Fuente: Felaban (2018)

Elaboración propia

Se calcula que existe una correlación negativa elevada entre la morosidad y la rentabilidad. A mayor morosidad, menor rentabilidad. Considerando 132 observaciones (desde enero del 2007 hasta diciembre del 2017), la correlación entre morosidad y rentabilidad sobre el patrimonio fue del $-0,9126$.

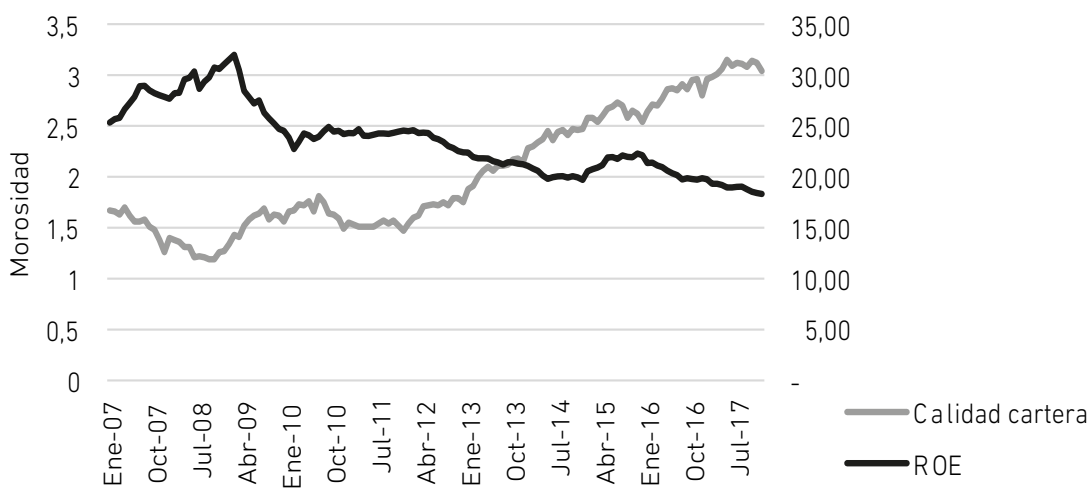

Figura 10. Evolución del ROE y la calidad de la cartera de la BM peruana

Fuente: SBS (2018b)

Elaboración propia 
Se visualizó el aumento de la morosidad a través del tiempo con la respectiva disminución de la rentabilidad sobre el patrimonio de la banca múltiple peruana. Sin embargo, la rentabilidad continuaba siendo una de las más altas de la región.

Durante el 2015 el deterioro de la cartera desaceleró su tendencia creciente, registrando una morosidad del 2,54 \%, lo que significa 7 bps (basis points) por encima del 2014, período durante el cual la morosidad aumentó 33 bps (basis points) con respecto al año 2013. Tal corrección se registró principalmente en la morosidad de los bancos retail en respuesta al deterioro de la cartera, las entidades bancarias tomaron acción en los procesos de recuperación de los créditos mostrando mayor rapidez en la activación de los procesos judiciales de la cartera vencida y presión por parte de las agencias de cobranza coactiva con el fin de aumentar la probabilidad de recuperación. Por lo que la cartera judicial aumentó paralelamente en los créditos refinanciados.

Más del $40 \%$ de los créditos fueron otorgados a las corporaciones y grandes empresas, registrando una morosidad promedio del 0,42 \%. Más del $20 \%$ de los créditos se otorgaron a micro, pequeñas y medianas empresas. Este tipo de crédito mostró un importante deterioro, pasando en el 2010 de un promedio del 2,98 \% a 7,35 \% de morosidad en el 2017. La morosidad más alta se registró en la pequeña empresa alcanzando hasta el $9 \%$.

En cuanto a los créditos de consumo, estos representaron aproximadamente el $18 \%$ de la cartera de créditos de la BM peruana. La morosidad de los créditos de consumo registró un promedio del 3,04 \% desde el 2007. Durante los años 2012 y 2013 hubo mayor disminución en la calidad de la cartera de este tipo de crédito, registrando variaciones de hasta dos dígitos.

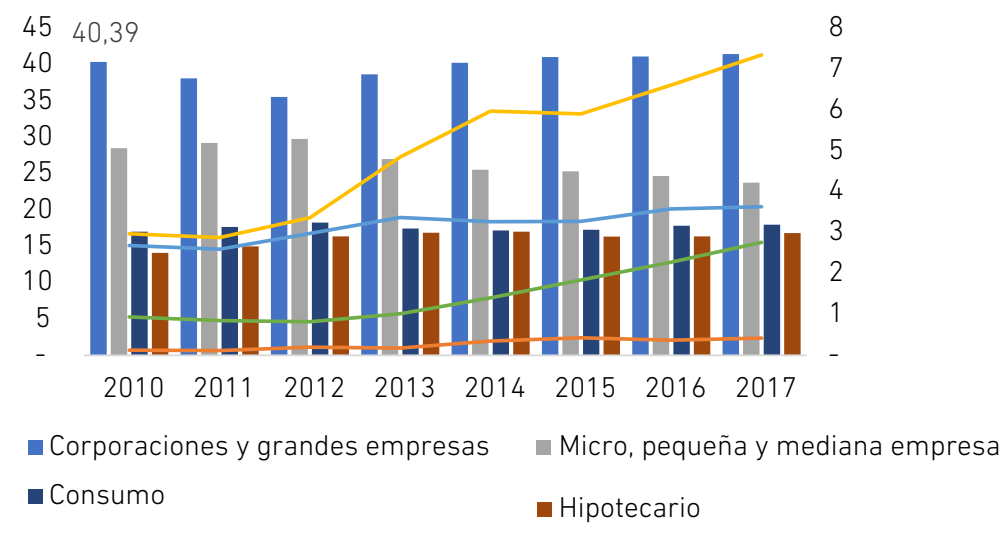

Figura 11. BM Perú. Estructura de créditos por tipo y evolución de la morosidad Nota: Información de años previos no comparable

Fuente: SBS (2018c)

Elaboración propia 
En cuanto a los créditos hipotecarios, en el año 2007 representaban el $12 \%$ de los créditos directos aumentando significativamente su participación en el mercado hasta representar el $17 \%$ en el 2017. Sin embargo, la morosidad registrada pasó del 0,94 \% al 2,76 \% durante el período. Dicho deterioro se presentó, principalmente, desde el año 2013.

Una de las principales causas de la morosidad está en la inestabilidad monetaria y la tendencia al alza del tipo de cambio, generándoles un alto riesgo para los deudores en dólares americanos.

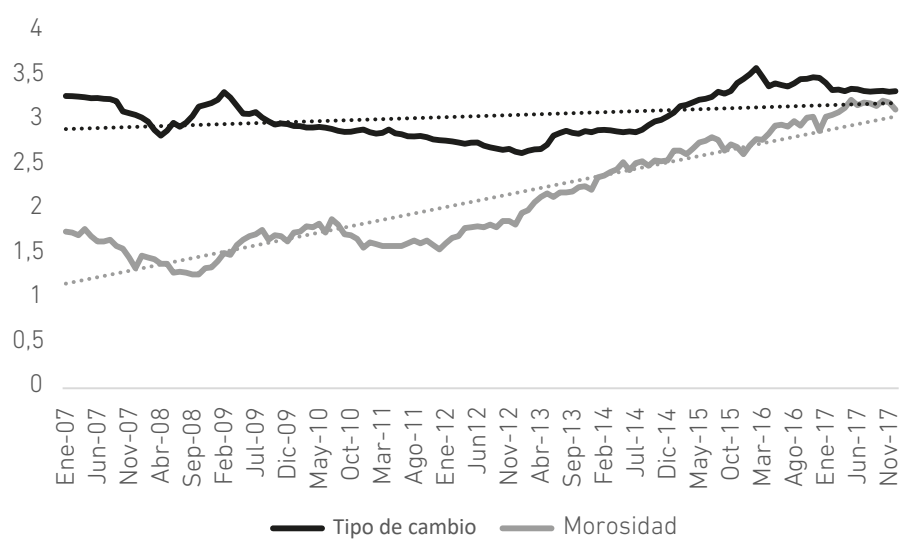

Figura 12. Perú. Evolución del tipo de cambio bancario promedio (soles por dólares americanos) versus morosidad

Fuente: BCRP (2018) y SBS (2018b)

Elaboración propia

La correlación entre el tipo de cambio y la morosidad es positiva. Durante el período 2007-2017 se calculó 0,5718 de correlación entre ambas variables, denotando que a mayor devaluación de la moneda se deteriorará la cartera.

\subsection{Cobertura}

A diciembre del 2017, la cobertura de la cartera fue de 152,61\%. Sin embargo, la tendencia de la cobertura fue decreciente. 


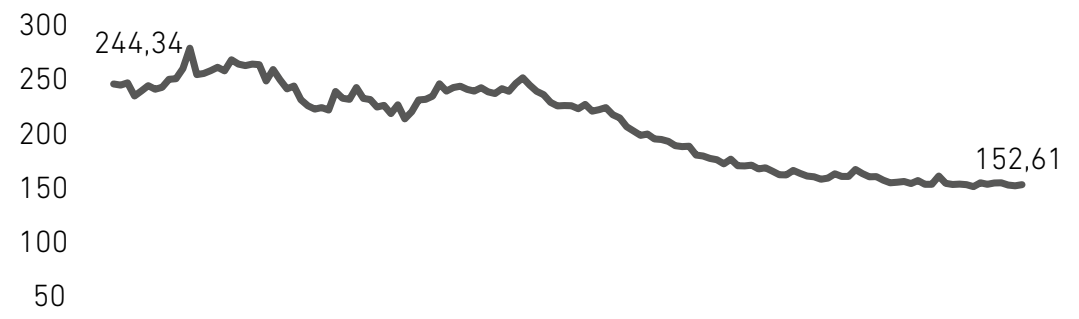

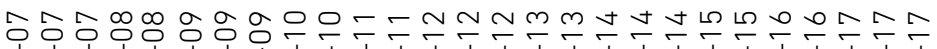

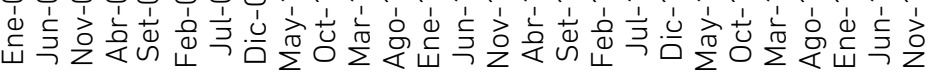

Figura 13. Cobertura de la BM peruana. Enero 2007 - diciembre 2017

Fuente: SBS (2018)

Elaboración propia

\subsection{Eficiencia}

Una mayor eficiencia implica mayores colocaciones y depósitos por cada sol de recursos consumidos. La BM en el Perú mostró una mayor eficiencia a lo largo del período de estudio. Por cada activo rentable, los gastos de administración fueron menores, y por cada sol de margen financiero, la BM registró menores gastos operativos.

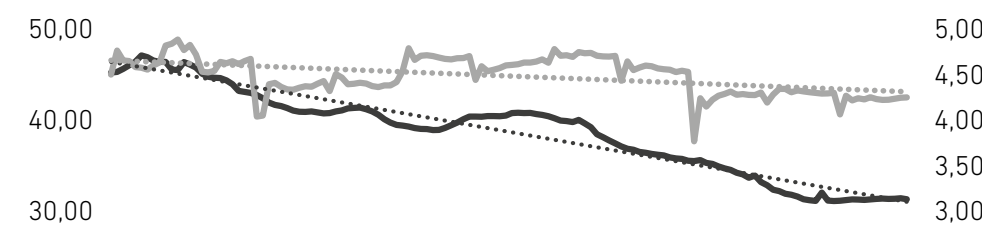

Gastos de administración anualizados / activo rentable promedio - Gastos de operación / margen financiero total

- Lineal (Gastos de administración anualizados / activo rentable promedio) - Lineal (Gastos de operación / margen financiero total)

Figura 14. BM Perú. Eficiencia en gastos de administración y de operación. Enero del 2007 a diciembre del 2017

Fuente: BCRP (2018)

Elaboración propia 
Asimismo, los créditos directos colocados por personal bancario y los depósitos captados por oficina mostraron una tendencia positiva durante el período de estudio.

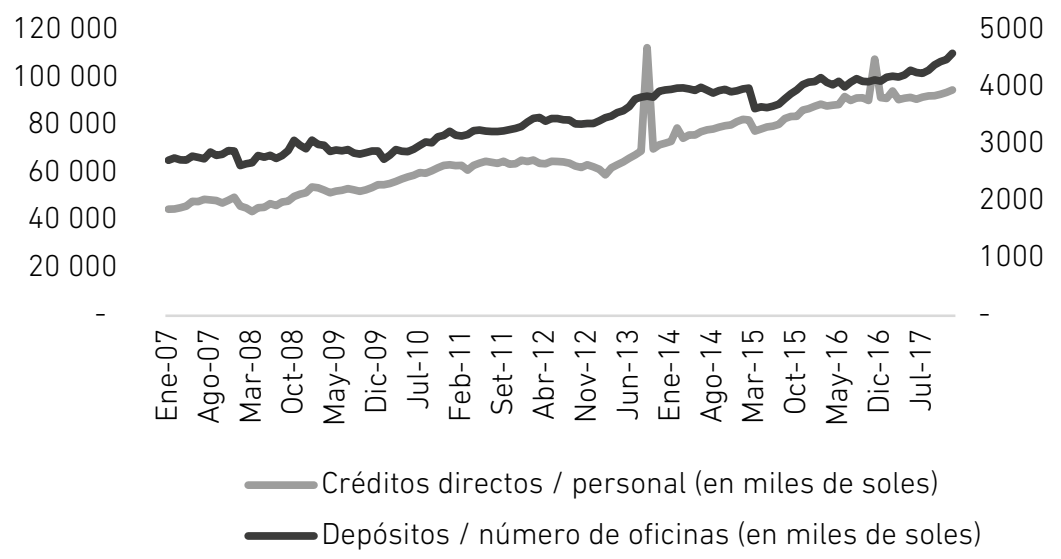

Figura 15. BM Perú. Eficiencia en personal y número de oficinas durante enero del 2007 y 2017

Fuente: BCRP (2018)

Elaboración propia

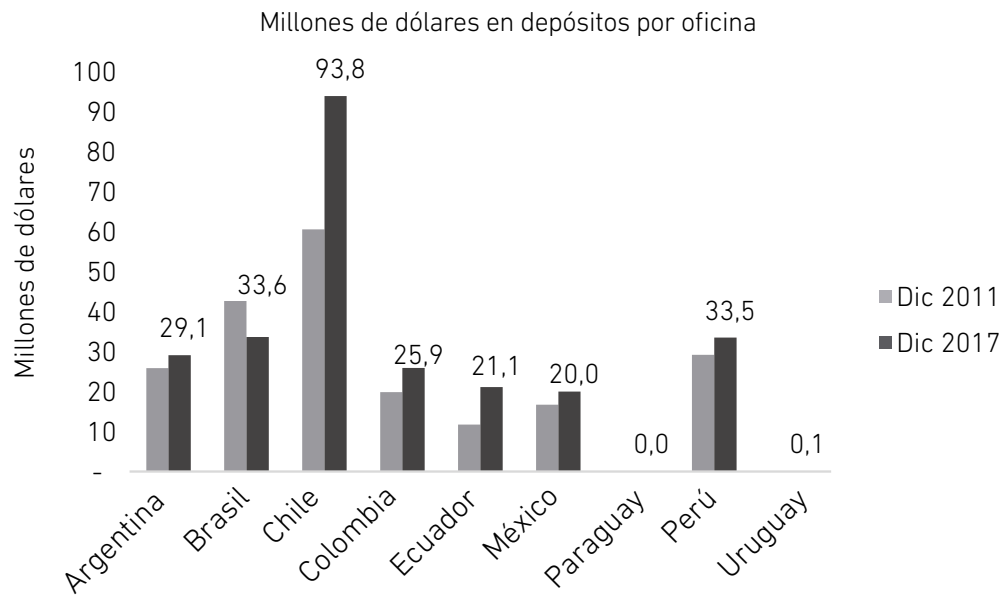




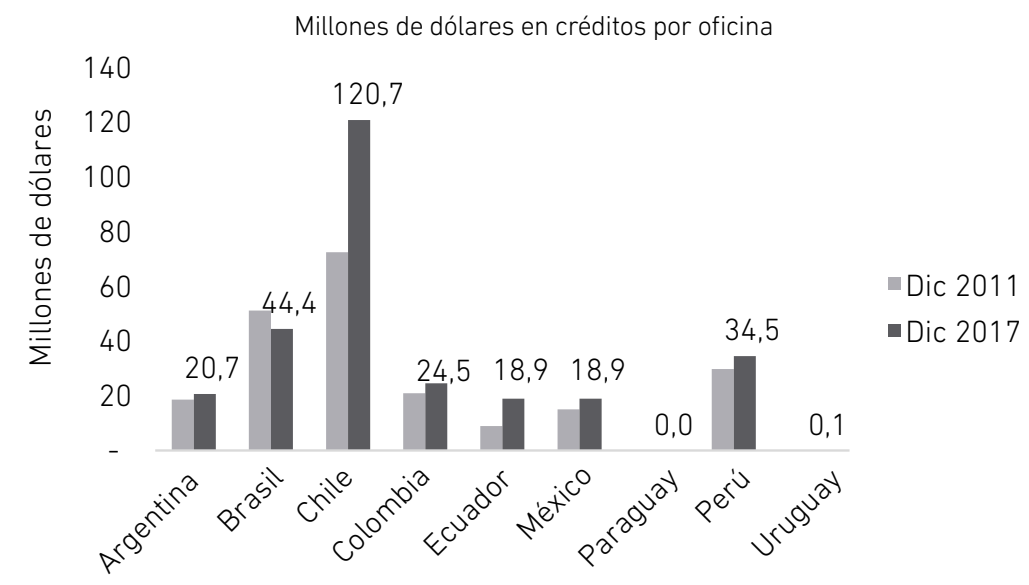

Figura 16. Créditos y depósitos por oficina en el 2011 versus 2017 en Latinoamérica Fuente: Felaban (2018)

Elaboración propia

\subsection{Rentabilidad}

La rentabilidad de la BM peruana a diciembre 2017 fue 18,32 (ROE) y 2,08 (ROA). La rentabilidad de la BM peruana dismimuyó desde el año 2009. A diciembre del 2008, la BM peruana registró la rentabilidad más alta con $31 \%$ de utilidades netas sobre el patrimonio. A finales del 2009 el ROE registrado fue $24,5 \%$ disminuyendo paulatinamente hasta $18,32 \%$ a diciembre 2017.

Sin embargo, la rentabilidad de la BM peruana continúa siendo la tercera rentabilidad más alta de los 9 países latinoamericanos considerados en el estudio a diciembre 2017, luego de Argentina (19\%) y Paraguay (18 \%). Los 6 países restantes mantienen una rentabilidad promedio del $12 \%$. La tendencia del promedio del ROE de la BM de los 9 países latinoamericanos en estudio y el ROE de la BM del Perú mostraron una tendencia decreciente a lo largo del tiempo. 
ROE promedio Latinoamérica

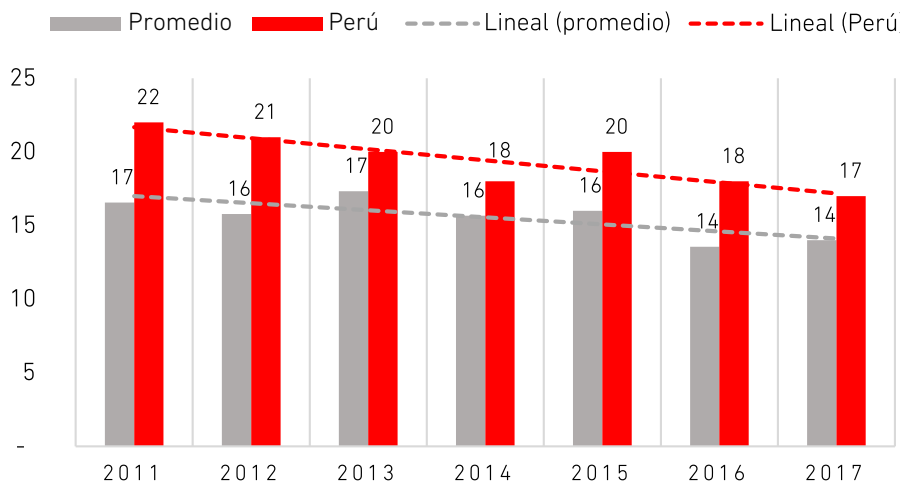

ROE Latinoamérica
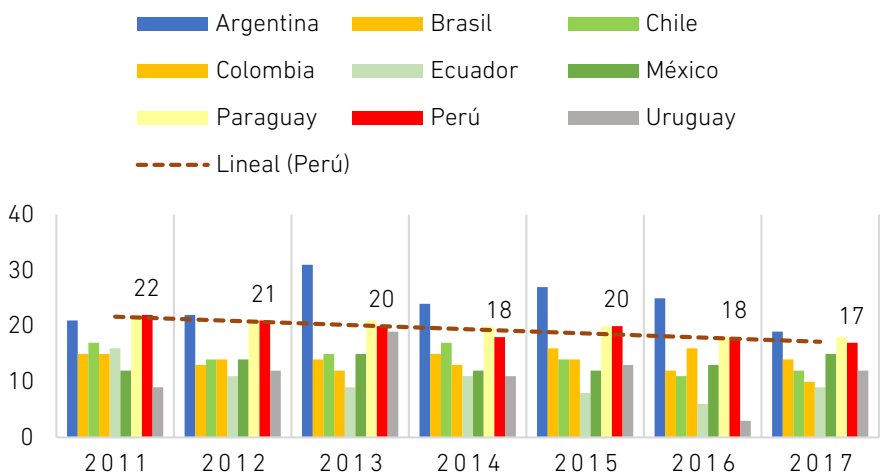

Figura 17. ROE de la BM del Perú y ROE promedio de BM de Latinoamérica (2011-2017)

Fuente: Felaban (2018)

Elaboración propia

La rentabilidad sobre los activos (ROA) durante el período de estudio mantuvo la tendencia decreciente del ROE. 


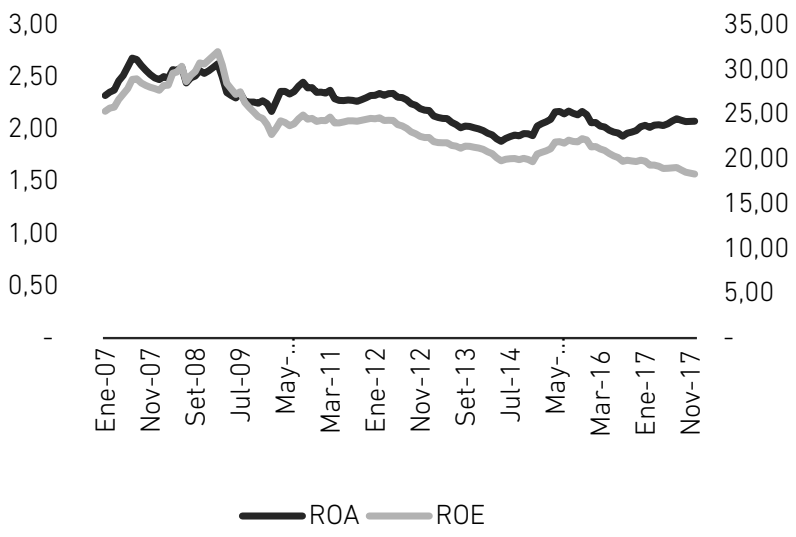

Figura 18. BM Perú. Evolución del ROE y ROA. Enero 2007-diciembre 2017

Fuente: SBS (2018)

Elaboración propia

Se encontró una alta correlación negativa entre el índice de precios al consumidor y la rentabilidad (ROE y ROA) durante el período de estudio (enero 2007-diciembre 2017). Mientras que la relación entre la variación porcentual del PBI y la rentabilidad de la BM en el Perú fue positiva, lo que significa que, a mayor crecimiento del producto bruto interno, mayor será la rentabilidad de la BM. Resulta que fue el crecimiento de las actividades no primarias las que están altamente correlacionadas con la rentabilidad de la BM peruana. Las actividades primarias no se relacionan con un crecimiento de la rentabilidad. Los resultados fueron los siguientes:

Tabla 3

Resultados de correlación entre variables macroeconómicas y la rentabilidad

\begin{tabular}{lcc}
\hline & ROE & ROA \\
\hline IPC & $-0,8675$ & $-0,8382$ \\
Variación porcentual PBI & $+0,4373$ & $+0,5654$ \\
Variación porcentual sector primario & $+0,0306$ & $+0,0030$ \\
Variación porcentual sectores no primarios & $+0,4705$ & $+0,6262$ \\
\hline
\end{tabular}

Elaboración propia

\subsection{Margen financiero}

El margen financiero bancario desde el año 2007 mostró una tendencia decreciente tanto en moneda nacional como en moneda extranjera. En el año 2007, la diferencia entre tasas 
activas y pasivas en moneda nacional fue del $20 \%$, mientras que, a diciembre 2017 , fue del 13,33 \%. En moneda extranjera, la variación no es tan pronunciada, pasando del 8,57 \% de spread (margen financiero), a enero 2007, al 6,18 \% a diciembre 2017. La principal reducción del spread bancario en moneda nacional sucedió durante el año 2013 con una variación negativa del casi $20 \%$, pasando en un solo año del $17 \%$ al 13,6 \%. El margen en moneda nacional se ha mantenido a ese nivel en promedio hasta la fecha.

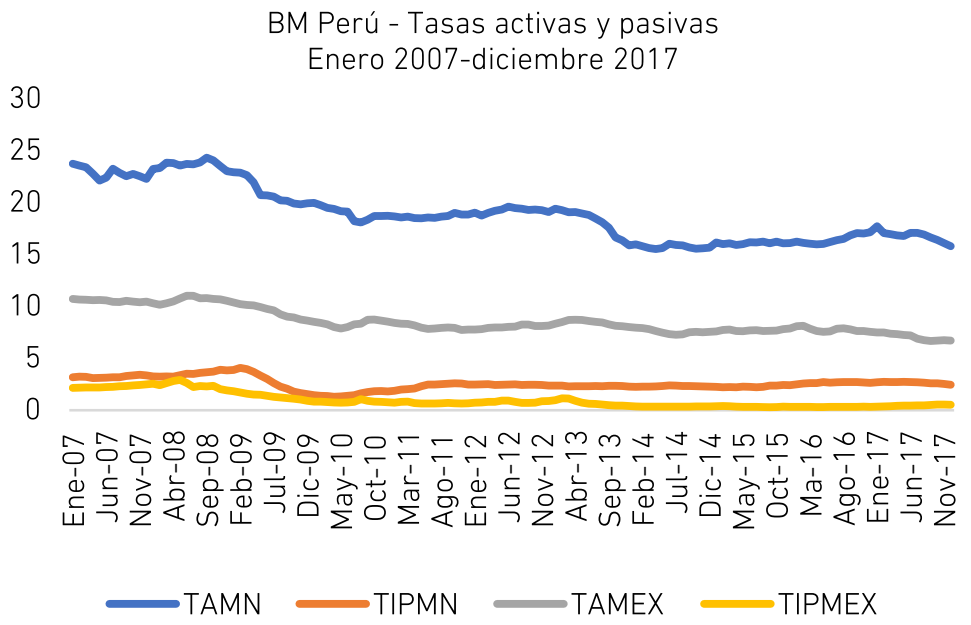

BM Perú - Spread

Enero 2007 - diciembre 2017

25

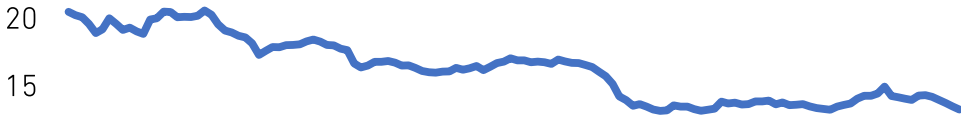

10

5

0

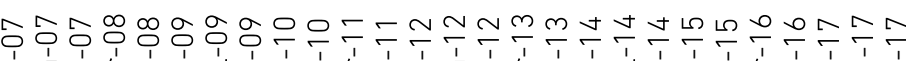

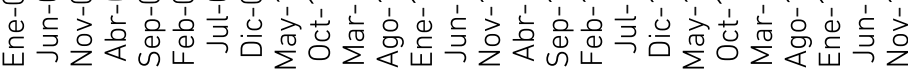
$\longrightarrow \mathrm{MN} \longrightarrow \mathrm{ME}$

Figura 19. Tasas activas y pasivas y margen financiero de la BM peruana Fuente: BCRP (2018)

Elaboración propia 


\subsection{Liquidez}

Durante el período de estudio, la liquidez de la BM peruana en soles mostró una tendencia decreciente y una tendencia creciente en moneda extranjera.

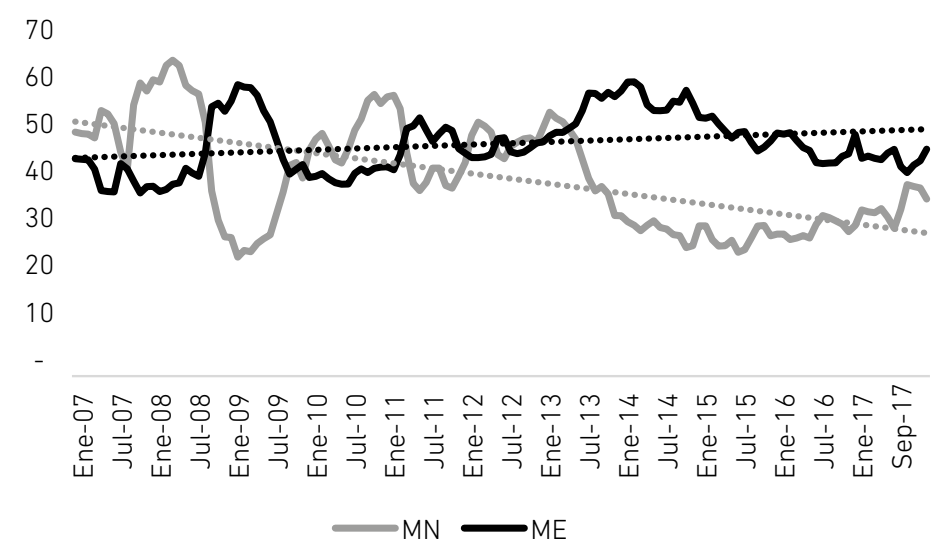

Figura 20. Evolución de la liquidez en moneda nacional (MN) y moneda extranjera (ME) durante 2007-2017

Fuente: SBS (2018)

Elaboración propia

El coeficiente de dolarización de la liquidez, tanto del sistema financiero como de la banca múltiple peruana, evidenció una tendencia decreciente. La liquidez de la BM peruana mantuvo un índice de dolarización por encima del sistema financiero. En enero 2007, el índice de dolarización de la BM era del 63,05\%, mientras que a diciembre 2017 este fue del 44,37 \%. El sistema financiero pasó del 33,79 \% al 23,50 \% a diciembre 2017.

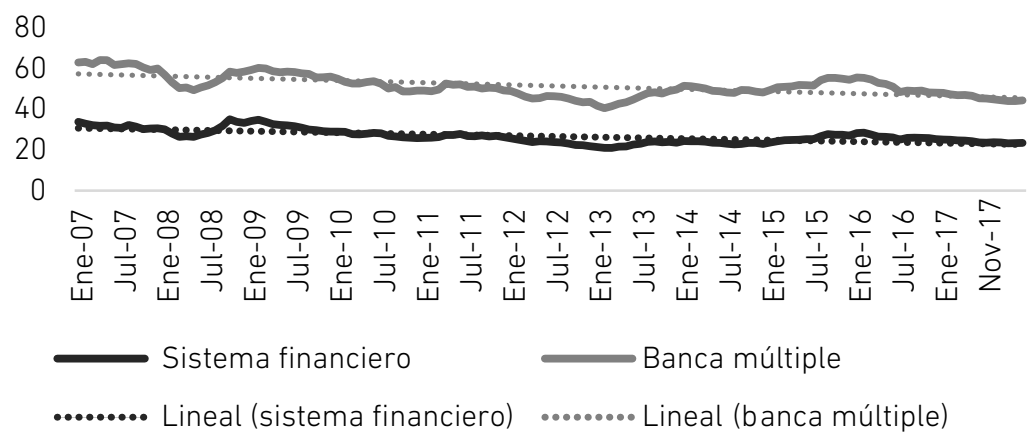

Figura 21. BM Perú. Coeficiente de dolarización de la liquidez

Fuente: BCRP (2018)

Elaboración propia 


\section{DISCUSIÓN Y CONCLUSIONES}

El sistema financiero peruano es uno de los sistemas con mayor concentración bancaria de Latinoamérica, la cual es afectada significativamente por las fusiones, adquisiciones, entradas y salidas de participantes con alto número de activos en el mercado. Períodos donde pequeños bancos ingresan o se retiran del mercado mantienen la concentración bancaria relativamente estable.

El patrimonio total de la BM en el Perú aumentó desde el año 2007. El patrimonio del sector se fortaleció acompañado de un crecimiento importante de las colocaciones.

La banca múltiple peruana en los últimos once años logró triplicar el monto de los activos, siendo su volumen comparable con países como Argentina y Colombia. Dada la alta correlación negativa entre la morosidad y la rentabilidad, se observó en la última década el aumento de la morosidad y la respectiva disminución de la rentabilidad sobre el patrimonio de la banca múltiple peruana.

La pobre infraestructura vial y de comunicaciones como la alta centralización de las actividades económicas en Lima, resultaron en altos costos de transacciones y bajo acceso al sistema financiero. Como consecuencia de lo anterior, es que el $85 \%$ de los depósitos y el 77 \% de los créditos tuvieron lugar en Lima, la capital. La centralización de las actividades económicas y políticas del Perú y el limitado alcance de los servicios repercutió negativamente en la eficiencia de la BM a nivel nacional, dificultando el crecimiento de la bancarización e inclusión financiera.

La BM peruana reflejó una tendencia decreciente de rentabilidad en los últimos años, al igual que la rentabilidad promedio de los nueve países latinoamericanos comprendidos en el análisis. El margen financiero bancario desde el año 2007 evidenció una tendencia decreciente tanto en moneda nacional como en moneda extranjera. Sin embargo, la rentabilidad de la BM peruana es la tercera más alta del grupo.

Para aprovechar el potencial de crecimiento, las oportunidades de mejora de la BM en el Perú radicaron en la inclusión financiera, la bancarización, la eficiencia enfocada en productos simples y diversificados y las economías de escala. Los riesgos a los que se expuso el Perú radicaron, principalmente, en: corrupción, exposición a volatilidad de precios internacionales y fluctuación de la moneda y vulnerabilidad ante fenómenos climatológicos. Por ello la lucha contra la corrupción, prevención de desastres naturales y la desdolarización de la economía son temas álgidos para su desarrollo.

\section{REFERENCIAS}

Banco Central de Reserva del Perú. (2018). Estadísticas económicas (2007-2017). Recuperado de https://estadisticas.bcrp.gob.pe/estadisticas/series/ 
Equilibrium Clasificadora de Riesgos S.A. (2018). Análisis del sistema bancario peruano. Recuperado de http://www.equilibrium.com.pe/bcosperujun18.pdf

Federación Latinoamericana de Bancos. (2018). Estadísticas, indicadores homologados. Recuperado de http://www.sbs.gob.pe/app/stats_net/stats/EstadisticaBoletin Estadistico.aspx?p=1\#

Galarza, F. T., y Miranda, G. G. (1995). Intermediación financiera y crecimiento económico. Cuadernos de Economía, 14(23), 178-196. Recuperado de https://revistas. unal.edu.co/index.php/ceconomia/article/view/13824/20400

Gómez Alberca, R. A., Villanueva, G., y Erixon, C. (2018). Efecto de las políticas monetarias sobre los créditos bancarios en el Perú, 2004-2016 (tesis de grado). Universidad Nacional Pedro Ruiz Gallo, Lambayeque, Perú. Recuperada de http://repositorio. unprg.edu.pe/handle/UNPRG/1981

Hansen, N. J., y Sulla, O. (2013). El crecimiento del crédito en América Latina: desarrollo financiero o boom crediticio. Revista Estudios Económicos, 25(1), 51-80. Recuperado de http://www.bcrp.gob.pe/docs/Publicaciones/Revista-EstudiosEconomicos/25/ree-25-hansen-sulla.pdf

Jeanneau, S., Tovar, C., y Moreno, R. (2007). Evolución de los sistemas bancarios en América Latina y el Caribe: retos e implicaciones para la política monetaria y la estabilidad financiera. BIS Papers, 33. Recuperado de http://www.bis.org/publ/ bppdf/bispap33_es.pdf

Levine, R. (2005). Finance and growth: theory and evidence. Handbook of economic growth, 1, 865-934.

Superintendencia de Banca, Seguros y AFP. (2018a). Estados financieros. Información de la banca múltiple. Recuperado de http://www.sbs.gob.pe/app/stats_net/stats/ EstadisticaBoletinEstadistico.aspx?p=1\#

Superintendencia de Banca, Seguros y AFP. (2018b). Indicadores financieros. Información de la banca múltiple. Recuperado de http://www.sbs.gob.pe/app/stats_net/ stats/EstadisticaBoletinEstadistico.aspx?p=1\#

Superintendencia de Banca, Seguros y AFP. (2018c). Morosidad por tipo de crédito y modalidad. Información de la banca múltiple. Recuperado de http://www.sbs.gob. pe/app/stats_net/stats/EstadisticaBoletinEstadistico.aspx?p=1\# 